\title{
Sciences cognitives et traditions d'enseignement oral de la musique classique indienne
}

The cognitive sciences and traditions of oral teaching in Indian classical music Ciencias cognitivas y tradiciones de enseñanza oral en la música clásica india

\section{Shantala Hegde}

Traducteur : Sylvaine Herold

\section{CpenEdition} Journals

Édition électronique

URL : https://journals.openedition.org/ries/5957

DOI : 10.4000/ries.5957

ISSN : 2261-4265

Éditeur

France Education international

\section{Édition imprimée}

Date de publication : 1 septembre 2017

Pagination : 75-85

ISBN : 978-2-85420-615-9

ISSN : $1254-4590$

Référence électronique

Shantala Hegde, «Sciences cognitives et traditions d'enseignement oral de la musique classique indienne », Revue internationale d'éducation de Sèvres [En ligne], 75 | septembre 2017, mis en ligne le 01 septembre 2019, consulté le 24 juin 2021. URL : http://journals.openedition.org/ries/5957 ; DOI : https://doi.org/10.4000/ries.5957 
alternative de quête spirituelle, destinée à aider les musiciens comme les auditeurs à accomplir leur voyage spirituel. Il semble aujourd'hui nécessaire de mener des recherches plus poussées sur la musique classique indienne, à partir des perspectives scientifiques actuelles, tant psychologiques que neurocognitives. Je conclurai cet article en citant un Doha (couplet) de Saint Kabir :

Guru so aisachaahiye, sheesseykachunalayei,

Shees so aisachaahiye, Guru ko sab kuchdayei

[Le guru idéal ne doit rien attendre en retour de son enseignement au disciple. Le disciple idéal doit vouloir tout donner au guru et se dévouer à lui.]

\section{BIBLIOGRAPHIE}

BHATKANDE V. (1934): Hindusthani Sangeet Paddhati, Sangeet Karyalaya.

BALKWILL L. L., THOMPSON W. F. (1999) : «A cross-cultural investigation of the perception of emotion in music: Psychophysical and cultural cues ", Music Perception, $\mathrm{n}^{\circ} 17$, p. 43-64.

EBYL. T., ALLEN T D., EVANS S C., NG T. et DUBOIS D. (2008) : « Does Mentoring Matter? A Multidisciplinary Meta-Analysis Comparing Mentored and Non-Mentored Individuals ", Journal of vocational behavior, $n^{\circ}$ 72(2), p. 254-267.

FERRERI L. (2017) : "Musique et plasticité cérébrale ", Revue internationale d'éducation de Sèvres, $\mathrm{n}^{\circ} 75$, p. 55-63.

HARTZELL J. F., DAVIS B., MELCHER D., MICELI G., JOVICICH J., NATH T., et al. (2016): "Brains of verbal memory specialists show anatomical differences in language, memory and visual systems ", Neuroimage, $\mathrm{n}^{\circ} 131$, p. 181-192.

HEGDE S. (2017) : "Music therapy for mental disorder and mental health: the untapped potential of Indian classical music », BJPsych International, 14(2) : 31-3.

HEGDE S., AUCOUTURIER J. J., RAMANUJAM B., BIGAND E. (2012) : «Variations in emotional experience during phases of elaboration of North Indian Raga performance ", actes de la $12^{\mathrm{e}}$ conférence internationale sur la perception et la cognition musicales et de la $8^{\mathrm{e}}$ conférence triennale des sciences cognitives de la musique, 23-28 juillet 2012, Thessalonique, Grèce, in E. Cambourpoulos, C. Tsougras, P. Mavromatis et K. Pastiadis (Eds.), p. 412-143.

JAIRAZBHOY N. A. (1995): The Ragas of North Indian Music: Their Structure and Evolution. Popular Prakashan Pvt Ltd.

KALAMANGALAM G. P., ELLMORE T. M. (2014) : «Focal cortical thickness correlates of exceptional memory training in Vedic priests ", Frontiers in Human Neuroscience, $\mathrm{n}^{\circ}$ 8, p. 833 .

KALYANI B. G., VENKATASUBRAMANIAN G., ARASAPPA R., RAO N.P., KALMADY S. V., BEHERE R. V. et al. (2011) : "Neurohemodynamic correlates of 'OM' chanting: A pilot functional magnetic resonance imaging study », International Journal of Yoga, $n^{\circ} 4(1)$, p. 3-6.

TRAINOR L. J., SHAHIN A J., ROBERTS L. E. (2009) : «Understanding the Benefits of Musical Training", Annals of the New York Academy of Sciences, 1169(1), p. 133-142. 